06

\title{
Анализ кинетики эволюции нанопор при отжиге субмикрокристаллических материалов
}

\author{
(C) В.Н. Перевезенцев, ${ }^{1,2}$ А.С. Пупынин, ${ }^{1,}$ А.Е. Огородников ${ }^{2}$ \\ ${ }^{1}$ Институт проблем машиностроения РАН, \\ 603024 Нижний Новгород, Россия \\ ${ }^{2}$ Нижегородский государственный университет им. Н.И. Лобачевского, \\ 603950 Нижний Новгород, Россия \\ ฯ e-mail: pupynin.as@gmail.com
}

(Поступило в Редакцию 29 декабря 2017 г.)

\begin{abstract}
Проанализирована кинетика эволюции нанопор, расположенных в тройных стыках зерен, в процессе низкотемпературного отжига субмикрокристаллических материалов, полученных методом интенсивной пластической деформации. Исследовано влияние на нее упругих напряжений от стыковых дисклинаций деформационного происхождения и неравновесности границ зерен.
\end{abstract}

DOI: 10.21883/JTF.2018.10.46498.2628

\section{Введение}

Многочисленные экспериментальные исследования свидетельствуют о том, что в субмикрокристаллических материалах, полученных методами интенсивной пластической деформации, на границах зерен и в их тройных стыках наблюдаются поры [1-8]. Широкий класс материалов, а также использование различных экспериментальных методик для исследования пористости позволяет говорить о том, что их образование при интенсивной пластической деформации носит закономерный характер. Микромеханизмы и условия зарождения нанопор в субмикрокристаллических материалах обсуждались в работе [9].

Формированием в процессе интенсивной пластической деформации нанопор в виде связной сетки полых нанотрубок, расположенных вдоль тройных стыков зерен, можно, по мнению авторов [4,5], объяснить экспериментальные данные, свидетельствующие, по крайней мере в ряде случаев, об аномально высоких скоростях диффузии в субмикрокристаллических материалах. В работе [6] увеличение неравновесного избыточного объема границ зерен в результате растворения зернограничных нанопор в процессе отжига и, как следствие, увеличение скорости зернограничной диффузии рассматривалось как возможная причина ускорения ползучести субмикрокристаллических сплавов, подвергнутых предварительному низкотемпературному отжигу. В работах В.И. Бетехтина и соавторов $[7,8]$ показано, что исходная пористость оказывает существенное влияние на упругие свойства субмикрокристаллических материалов, а также на их долговечность в условиях длительного нагружения.

Теоретически кинетика эволюции пор при отжиге была исследована в работе [10]. Позднее предложенная авторами этой работы модель была модифицирована для анализа кинетики роста и растворения нанопор в процессе отжига субмикрокристаллических материалов [4].
В упомянутых работах, однако, при построении моделей растворения пор не учитывалось влияние на кинетику этого процесса внутренних напряжений от мезодефектов, возникающих в процессе интенсивной пластической деформации на границах и в стыках зерен [11], а также влияние на нее изменяющегося в процессе отжига неравновесного состояния границ зерен. В настоящей работе предпринята попытка проанализировать влияние этих факторов на кинетику растворения нанопор в процессе низкотемпературного отжига субмикрокристаллических материалов.

\section{1. Описание модели}

Вследствие различия кристаллографических ориентаций зерен пластическая деформация по ансамблю зерен поликристалла протекает неоднородно. Неодинаковость пластической деформации соседних зерен приводит к появлению на разделяющей их границе зерна дополнительных разориентировок $\Delta \Theta_{i}[12]$ :

$$
\Delta \Theta_{i}=\mathbf{N}_{i} \times[\Delta \hat{\varepsilon}] \mathbf{N}_{i},
$$

где $\mathbf{N}_{i}$ - вектор нормали к плоскости $i$-ой границы зерна, а величина $[\Delta \hat{\varepsilon}]$ представляет собой скачок тензора пластической деформации при переходе через $i$-ую границу зерна. В результате рассогласования разориентировок границ в тройных стыках зерен возникают линейные мезодефекты ротационного типа - стыковые дисклинации деформационного происхождения (СД ДП) с мощностью $\omega=\sum \Delta \Theta_{i},(i=1,2,3)$, создающие внутренние упругие напряжения $\sigma_{i} \approx G \omega / 2 \pi(1-v)(G-$ модуль сдвига, $v$ - коэффициент Пуассона) [13]. Как показывают результаты экспериментальных [14,15] и теоретических исследований [16], характерная мощность СД ДП составляет порядка $(1-2)^{\circ}$.

В силу случайного характера кристаллографической ориентации зерен и плоскостей залегания границ мощ- 
ность СД ДП в различных стыках зерен в общем случае должна быть различной, что существенно затрудняет аналитическое описание. Для простоты в дальнейшем мы будем считать, что разброс величины $\omega$ по ансамблю тройных стыков поликристалла мал и в первом приближении им можно пренебречь. Ниже рассматривается модельный поликристалл, состоящий из одинаковых гексагональных зерен, в тройных стыках которого расположены чередующиеся по знаку СД ДП одинаковой мощности (рис. 1,a). Дефектная структура такого поликристалла представляет собой совокупность двуосных диполей клиновых дисклинаций. Эквивалентную ей по полям упругих напряжений дислокационную структуру можно представить как расположенные вдоль границ зерен стенки континуально распределенных краевых сидячих дислокаций с плотностью вектора Бюргерса, равной по величине мощности СД ДП (рис. $1, b$ ).

Рассматривались поликристаллы, содержащие в исходном состоянии поры двух видов - в виде вытянутых вдоль линии тройных стыков зерен полых трубок радиуса $r_{1}$ (рис. $\left.2, a\right)$ и в виде цепочки эквидистантно расположенных вдоль тех же стыков на расстоянии $\lambda_{p}$ сферических пор одинакового радиуса $r_{2}$ (рис. $2, b$ ). Сравнительный анализ поверхностных энергий соответству-
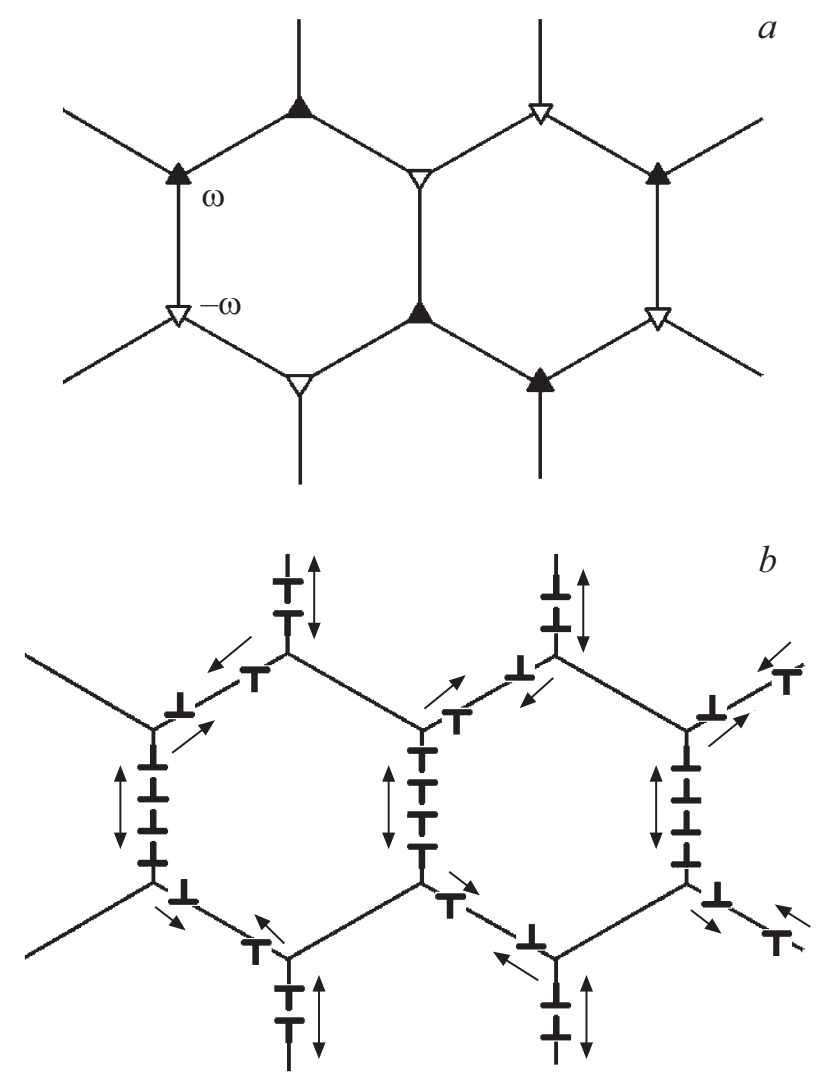

Рис. 1. Схематическое представление поликристалла с расположенными в тройных стыках зерен разноименными СД мощностью $\omega(a)$ и эквивалентная дислокационная схема для системы СД ДП $(b)$. Стрелками указаны направления переползания дислокаций.
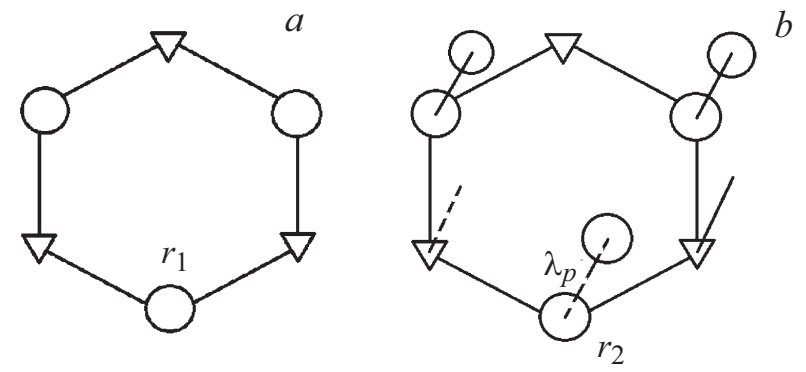

Рис. 2. Схематическое представление поликристалла с расположенными в тройных стыках порами: $a-$ поры в виде трубок радиуса $r_{1}$ и длины $d$ (вид сверху); $b-$ сферические поры радиуса $r_{2}$, расположенные друг от друга на расстоянии $\lambda_{p}$.

ющих конфигураций в рассматриваемом поликристалле показывает, что при одном и том же суммарном объеме пор второй случай оказывается более энергетически выгодным при выполнении условий $\lambda_{p} / r_{2}>3\left(\pi g_{s b}\right)^{2} / 4$ и $r_{2} / r_{1}>3 \pi g_{s b} / 4$, где $g_{s b}=\left(4 \gamma_{s}-3 \gamma_{b} / 2\right) /\left(2 \pi \gamma_{s}-3 \gamma_{b}\right)$, $\gamma_{s}$ и $\gamma_{b}$ - удельные энергии свободной поверхности и границы зерна соответственно. Заметим, что для рассматриваемого модельного поликристалла в области тройных стыков, содержащих положительные СД ДП, действуют растягивающие напряжения, а в области тройных стыков, в которых расположены отрицательные СД ДП, создаются сжимающие напряжения [17]. Поэтому поры на рис. 2 расположены не во всех тройных стыках, а только в тех, где действуют стабилизирующие их растягивающие напряжения.

При анализе кинетики эволюции пористости учитывались изменение мощности СД ДП в процессе отжига и зависимость коэффициента зернограничной диффузии от неравновесного состояния границ зерен.

\section{1. Кинетика эволюции пористости при отжиге}

Рассмотрим кинетику эволюции размеров пор для конфигураций, представленных на рис. 2.

1.1.1. Цилиндрические поры Изменение объема поры при низкотемпературном отжиге, когда диффузией из объема в пору можно пренебречь, происходит путем короткозамкнутых (в области размером порядка размера зерна d) диффузионных потоков атомов из границы зерна в пору. Движущей силой массопереноса является градиент химических потенциалов атомов $\nabla \mu \approx\left(\mu_{b}-\mu_{s 1}\right) / d$, связанный с различием химических потенциалов зернограничных атомов

$$
\mu_{b}=\alpha_{d} G \omega \Omega_{a}+\frac{K_{b} \Omega_{a}}{2} \Delta f-k T \ln \left(1+\frac{\Delta f}{f^{*}}\right)
$$

и атомов на поверхности поры

$$
\mu_{s 1}=\gamma_{s} \Omega_{a} / r_{1} .
$$


Здесь $\alpha_{d} \approx 0.3, \Omega_{a}$ - объем атома, $K_{b}-$ коэффициент всестороннего сжатия, $\Delta f-$ избыточный относительный свободный объем неравновесной границы и $f^{*}$ - свободный объем равновесной границы зерна, $k$ - постоянная Больцмана, $T-$ температура отжига. В выражении (2) первое слагаемое описывает вклад в химический потенциал зернограничных атомов поля упругих напряжений от системы СД ДП, второе и третье слагаемое обусловлены неравновесным состоянием границ зерен. С учетом этого уравнение, описывающее кинетику изменения объема пор, в конечном итоге приобретает вид

$$
\begin{array}{r}
\dot{V}_{1}=\frac{3 \delta D_{b}}{k T}\left[\alpha_{d} G \omega \Omega_{a}+\frac{K_{b} \Omega_{a}}{2} \Delta f\right. \\
\left.-k T \ln \left(1+\frac{\Delta f}{f^{*}}\right)-\gamma_{s} \Omega_{a} / r_{1}\right],
\end{array}
$$

где $\delta=2 b$ - ширина границы зерна, $b$ - вектор Бюргерса, $D_{b}$ - коэффициент зернограничной диффузии.

1.1.2. Сферические поры Пусть сферические поры радиусом $r_{2}$ расположены вдоль тройных стыков поликристалла на расстоянии $\lambda_{p}$ друг от друга (рис. $2, b$ ). В рассматриваемой модели предполагается отсутствие взаимодействия между порами в соседних стыках. Аналогичное уравнение для скорости изменения объема сферической поры приобретает вид

$$
\begin{aligned}
\dot{V}_{2} & =\frac{3 \pi \delta D_{b}}{k T} \frac{r_{2}}{d}\left[\alpha_{d} G \omega \Omega_{a}+\frac{K_{b} \Omega_{a}}{2} \Delta f\right. \\
& \left.-k T \ln \left(1+\frac{\Delta f}{f^{*}}\right)-2 \gamma_{s} \Omega_{a} / r_{2}\right],
\end{aligned}
$$

где учтено, что для сферической поры величина $\mu_{s 2}=2 \gamma_{s} \Omega_{a} / r_{2}$.

\section{2. Кинетика изменения мощности стыковых дисклинаций деформационного происхождения}

Протекание процесса возврата при отжиге, приводящего к уменьшению мощности СД ДП $\omega$, в эквивалентной структуре, показанной на рис. $1, b$, можно описать как переползание дислокаций с их последующей аннигиляцией с дислокациями противоположного знака (на рис. $1, b$ стрелками указаны направления переползания дислокаций). При низкотемпературном отжиге скорость этого процесса лимитируется скоростью диффузионного массопереноса по границам зерен.

Кинетика изменения величины $\omega$ в рамках рассматриваемой модели определяется уравнением

$$
\dot{\omega}=-\beta \frac{G \Omega_{a}}{k T} \frac{\delta}{b} \frac{D_{b}}{d^{2}} \omega^{2}
$$

где $\beta$ - геометрический множитель порядка 1.

\section{3. Изменение неравновесного состояния границ зерен и коэффициента зернограничной диффузии в процессе отжига}

Степень неравновесности границ зерен принято характеризовать величиной относительного неравновесного свободного объема $\Delta f$ [18]. Согласно [18], связь между коэффициентом зернограничной диффузии $D_{b}$ и величиной $\Delta f$ имеет вид

$$
D_{b}=D_{b}^{*} \exp (\alpha \Delta f)
$$

где $D_{b}^{*}-$ коэффициент диффузии по равновесным границам [18], $\alpha=K_{b} \Omega_{a} / 2 k T$.

Кинетическое уравнение баланса для относительного неравновесного объема $\Delta f$ в случае растворения цилиндрических пор можно записать в виде

$$
\Delta \dot{f}=-\frac{\dot{V}_{1}}{3 \delta d^{2}}-\frac{2 \Omega_{a}}{\delta}\left[A_{0} \exp (\alpha \Delta f)-B C_{v}(x=0)\right],
$$

где первое слагаемое описывает изменение величины $\Delta f$ в процессе растворения нанопор, а второе слагаемое процессы обмена вакансиями между границей и объемом зерен $[19,20]$. Для случая сферических пор изменится только вид первого слагаемого

$$
\Delta \dot{f}=-\frac{\dot{V}_{2}}{3 \lambda_{p} \delta d}-\frac{2 \Omega_{a}}{\delta}\left[A_{0} \exp (\alpha \Delta f)-B C_{v}(x=0)\right]
$$

Здесь $[19,20] A_{0}=\omega_{b} / b^{2} \exp \left(-\left[F_{v f}^{c r}+\Delta F+0.5 K_{b} \Omega_{a} f^{*}\right] /\right.$ $/ k T), F_{v f}^{c r}-$ свободная энергия образования решеточной вакансии, $\Delta F-$ свободная энергия активации перескока атома из объема зерна в зернограничную вакансию, $B=b \omega_{c} \exp (-\Delta F / k T), \omega_{b}$ и $\omega_{c}-$ частота колебаний атомов в границе зерна и в кристаллической решетке соответственно, $C_{v}(x=0)-$ концентрация вакансий вблизи границы зерна.

Уравнения (4)-(9), дополненные стандартным уравнением объемной диффузии вакансий с граничными условиями, приведенными в работах $[19,20]$, образуют замкнутую систему, позволяющую проанализировать влияние различных факторов (внутренних напряжений, степени неравновесности границ зерен, размера зерна) на кинетику эволюции пор при отжиге.

\section{2. Результаты численных расчетов и обсуждение}

Численное расчеты проводилось для случая низкотемпературного отжига $\left(T=0.4 T_{m}\right)$ субмикрокристаллического алюминия с размером зерна $d=$ $=4 \cdot 10^{-7} \mathrm{~m}$ при следующих значениях параметров: $b=2.86 \cdot 10^{-10} \mathrm{~m}, \quad \Omega_{a}=b^{3}, \quad \omega_{b} \approx \omega_{c}=10^{13} \mathrm{~s}^{-1}$, $\lambda_{p}=32 \mathrm{~nm}, \quad D_{b}=1.5 \cdot 10^{-16} \mathrm{~m}^{2} / \mathrm{s} \quad[21], \quad H_{v f}^{c r}=10 k T_{m}$, $S_{v f}^{c r}=4 k, \Delta F=8 k T_{m}[22]$. 

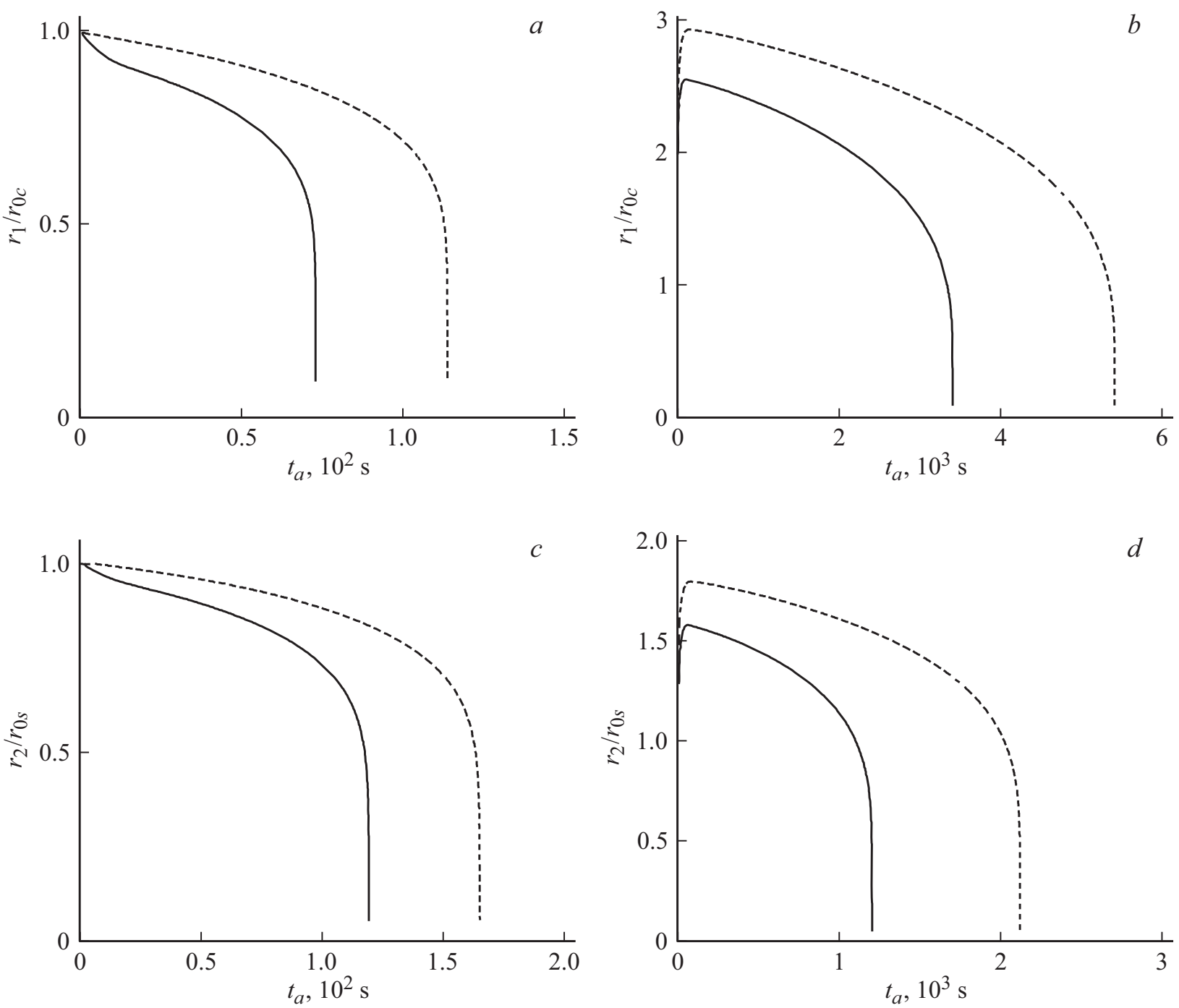

Рис. 3. Зависимости радиусов цилиндрических $r_{1}(a, b)$ и сферических $r_{2}(c, d)$ пор от времени отжига $t_{a}$ при значениях мощности стыковых дисклинаций $\omega_{1}=1^{\circ}$ (сплошные линии) и $\omega_{2}=2^{\circ}$ (штриховые линии) при различной исходной степени неравновесности границ зерен $\Delta f_{0}=0(a, c), \Delta f_{0}=0.015(b, d) . r_{0 c}=3 \mathrm{~nm}, r_{0 s}=6 \mathrm{~nm}$.

Результаты расчетов эволюции пор представлены на рис. 3. Приведены зависимости радиусов цилиндрических $r_{1}$ (рис. $\left.3, a, b\right)$ и сферических $r_{2}$ (рис. $3, c, d$ ) пор от времени отжига $t_{a}$, полученные при различных значениях исходной мощности СД ДП $\left(\omega_{0}\right)$ и различной степени исходной неравновесности границ зерен $\left(\Delta f_{0}\right)$. Видно, что увеличение исходной мощности СД ДП приводит, как и следовало ожидать, к существенному замедлению процесса растворения пор. Менее очевидным представляется влияние на этот процесс исходной степени неравновесности границ зерен. Как видно из рис. $3, b, d$, при $\Delta f_{0}=0.015$ на начальном этапе отжига наблюдается не растворение пор, а их рост, кроме того, по сравнению с исходно равновесными границами зерен $\left(\Delta f_{0}=0\right)$ время полного растворения пор увеличивается. Указанные особенности растворения пор тем не менее легко объяснимы. Рост пор на начальном этапе отжига при наличии исходной неравно- весности границ зерен связан со стоком неравновесных зернограничных вакансий в поры. Одновременно со стоком неравновесных вакансий в поры происходит и их отток в объем зерен. Это приводит к тому, что стадия роста пор оказывается довольно непродолжительной.

В заключение отметим, что в работе [4] ее авторы при теоретическом анализе кинетики эволюции нанопор учитывали изменение размера зерна в процессе отжига. В рамках рассмотренной выше модели мы пренебрегли ростом зерен исходя из тех соображений, что СД ДП тормозят процессы миграции границ и роста зерен [23]. Можно полагать, что рост зерен может оказаться существенным на поздних стадиях растворения пор вследствие уменьшения мощности и увеличения подвижности СД ДП в процессе возврата дефектной структуры. Рассмотрение кинетики эволюции пор с учетом возможного влияния роста зерен будет проведено в дальнейшем. 


\section{Выводы}

1. Установлено, что дисклинации, сформировавшиеся в стыках зерен поликристалла в процессе предварительной пластической деформации, существенно замедляют процесс растворения нанопор при низкотемпературном отжиге.

2. Показано, что увеличение исходной степени неравновесности границ зерен приводит к появлению непродолжительной стадии роста пор на начальном этапе низкотемпературного отжига, а также к увеличению времени полного растворения пор.

Работа выполнена в рамках государственного задания ИПФ РАН на проведение фундаментальных научных исследований на 2013-2020 гг. по теме № 0035-2014-0401.

\section{Список литературы}

[1] Бетехтин В.И., Табачникова Е.Д., Кадомцев А.Г., Нарыкова M.В., Lapovok R. // Письма в ЖТФ. 2011. Т. 37. Вып. 16. С. 52-55. [Betekhtin V.I., Tabachnikova E.D., Kadomtsev A.G., Narykova M.V., Lapovok R. // Tech. Phys. Lett. 2011. Vol. 37. N 8. P. 767-768.] DOI: $10.1134 / \mathrm{S} 1063785011080189$

[2] Бетехин В.И., Колобов Ю.Р., Нарыкова М.В., Кардашев Б.К., Голосов Е.В., Кадомиев А.Г. // ЖТФ. 2011. Т. 81. Вып. 11. С. 58-63. [Betekhtin V.I., Kolobov Yu.R., Narykova M.V., Kardashev B.K., Golosov E.V., Kadomtsev A.G.] // Tech. Phys. 2011. Vol. 56. N 11. P. 1599-1604]. DOI: https://doi.org/10.1134/S1063784211110053

[3] Dvorak J., Sklenička V., Betekhtin V.I., Kadomtsev A.G., Kral P., Kvapilova M., Svoboda M. // Mater. Sci. Eng. A. 2013. Vol. 584. P. 103-113.

DOI: https://doi.org/10.1016/j.msea.2013.07.018

[4] Ribbe J., Schmitz G., Gunderov D., Estrin Y., Amouyal Y., Wilde G., Divinski S.V. // Acta Mater. 2013. Vol. 61. P. 5477-5486. DOI: http://dx.doi.org/10.1016/j.actamat.2013.05.036

[5] Divinski S.V., Reglitz G., Golovin I.S., Peterlechner M., Lapovok R., Estrin Y., Wilde G. // Acta Mater. 2015. Vol. 82. P. 11-21. DOI: http://dx.doi.org/10.1016/j.actamat.2014.08.064

[6] Перевезенцев В.Н., Щербань М.Ю., Грачева Т.А., Кузьмичева Т.А. // ЖТФ. 2015. Т. 85. Вып. 8. С. 63-66. [Perevezentsev V.N., Shcherban' M.Yu., Gracheva T.A., Kuz'micheva T.A. // Tech. Phys. 2011. Vol. 60. N 8, P. 1167-1170] DOI: https://doi.org/10.1134/S1063784215080204

[7] Кардашев Б.К., Бетехтин В.И., Кадомиев А.Г., Нарыкова М.В., Колобов Ю.Р. // ЖТФ. 2017. Т. 87. Вып. 9. С. 1362 1366. [Kardashev B.K., Betekhtin V.I., Kadomtsev A.G., Narykova M.V., Kolobov Yu.R. // Tech. Phys. 2017. Vol. 62. N 9, P. 1372-1376.] DOI: https://doi.org/10.1134/S1063784217090110

[8] Бетехтин В.И., Sklenička V., Кадомцев А.Г., Колобов Ю.Р., Нарыкова М.В. // ФТТ. 2017. Т. 59. Вып. 5. С. 935-941. [Betekhtin V.I., Sklenička V., Kadomtsev A.G., Kolobov Yu.R., Narykova M.V. // Phys. Sol. Stat. 2017. Vol. 59. N 5. P. 960-966.] DOI: 10.21883/FTT.2017.05.44383.391
[9] Ovid'ko I. A., Sheinerman A.G., Skiba N.V. // Acta Mater. 2011. Vol. 59. P. 678-685.

DOI: $10.1016 /$ j.actamat.2010.10.005

[10] Klinger L., Rabkin E., Shvindlerman L.S., Gottstein G. // J. Mater. Sci. 2008. Vol. 43. P. 5068-5085. DOI: $10.1007 / \mathrm{s} 10853-008-2678-\mathrm{y}$

[11] Rybin V.V., Zisman A.A., Zolotorevsky N.Yu. // Acta Metall. Mater. 1993. Vol. 41. N 7. P. 2211-2217. DOI: https://doi.org/10.1016/0956-7151(93)90390-E

[12] Перевезенцев В.Н., Рыбин В.В. Структура и свойства границ зерен: Учебное пособие. Нижний Новгород: Изд-во Нижегородского гос. ун-та им. Н.И. Лобачевского, 2012. $307 \mathrm{c}$.

[13] Klemm V., Klimanek P., Kolesnikova A.L., Motylenko M., Romanov A.E. // Ann. Chim. Sci. Mater. 2002. Vol. 27. P. 25-33.

[14] Klimanek P., Klemm V., Romanov A.E., Seefeldt M. // Adv. Eng. Mater. 2001. Vol. 3. N 11. P. 877-884.

DOI: 10.1002/1527-2648(200111)3:11<877::AID-ADEM877>3.0.CO;2-L

[15] Klemm V., Klimanek P., Motylenko M. // Mater. Sci. Eng. A. Vol. 324. P. 174-178. DOI: https://doi.org/10.1016/S0921-5093(01)01307-7

[16] Nazarov A.A., Romanov A.E., Valiev R.Z. // Scr. Mater. 1996. Vol. 34. P. 729-734. DOI: https://doi.org/10.1016/13596462(95)00573-0

[17] Лихачев В.А., Хайров Р.Ю. Введение в теорию дисклинаций. Л.: Изд-во Ленинградского ун-та, 1975. $183 \mathrm{c}$.

[18] Перевезенцев В. Н. // ФММ. 2002. Т. 93. № 3. С. 15-19. [Perevezentsev V.N. // Phys. Metals Metallography. 2002. Vol. 93. N 3. P. 207-210.

[19] Перевезенцев В.Н., Пупынин А.С., Свирина Ю.В. // ФММ. 2005. T. 100. № 1. C. 17-23. [Perevezentsev V.N., Pupynin A.S., Svirina Yu.V. // Phys. Metal. Metallography. 2005. Vol. 100. N 1. P. 12-17.]

[20] Perevezentsev V. N., Pupynin A. S., Svirina J.V. // Mater. Sci. Eng. A. 2005. Vol. 410-411. P. 273-276. DOI: https://doi.org/10.1016/j.msea.2005.08.002

[21] Фрост Г.Джс., Эиби М.Ф. Карты механизмов деформации. / Пер. с англ. Под ред. Л.М. Берштейна. Челябинск: Металлургия, Челябинское отделение, 1989. 328 с.

[22] Перевезенцев В.Н., Пупынин А.С. // Письма в ЖТФ. 2011. T. 37. Вып. 6. C. 88-94. [Perevezentsev V.N., Pupynin A.S. // Tech. Phys. Lett. 2011. Vol. 37. N 3. P. 287-290.] DOI: https://doi.org/10.1134/S106378501103028X

[23] Чувильдеев В.Н. Неравновесные границы зерен в металлах. Теория и приложения. М.: Физматлит, 2004. 304 с. 